\title{
Comprehensive Visible Light Communication System for Healthcare
}

\author{
Shivani Singh, Sumita Mishra, Pallavi Asthana, Sachin Kumar \\ Department of Electronics and communication engineering, Amity University, Lucknow, UP
}

\begin{abstract}
Health care monitoring is becoming essential with the increase in high risk diseases among the people of all ages. Various wireless sensors are present which continuously monitor body parameters such as pulse rate, ECG etc and record them. Radio frequency communication is the largely accepted channel for the realization of patient health monitoring technology which presents enormous potential for future healthcare application. Visible Light Communication is an emerging technology that can be utilized for similar applications with an edge overradio-frequency, which suffers from multiple issues for example interference with medical instruments and in some cases cause hazards to the health of patient. Visible Light Communication uses LED as a transmitter and it can become a better alternate for the wireless data transmission in various healthcare systems. This paper presents in brief Visible light communication (VLC) for indoor medical applications.
\end{abstract}

Keywords: VLC, Healthcare, OOK modulation, LED.

\section{Introduction}

Enormous growth of mobile technologies has penetrated almost each and every aspect of human life. This technology has become an essential part of living. In the present scenario of increasing health issues, many of health monitoring devices have become a necessary part of livelihood. New generation healthcare systems focus on early detection of problems and their prevention by providing positive wellness management system [1]. Wearable and implantable Radio frequency (RF) based wireless health devices attracts more attention [2], to meet these requirements.

A lot of attention is focused on devices that can be used for healthcare system or for other applications in a controlled environment [3-5].Currently used devices are mainly based on wireless technologies such as Bluetooth [6], ZigBee [7] and advanced Bluetooth Low Energy (BLE) [8] with low power consumption.

Integration of these added mobility to healthcare monitoring systems and provided significant interest in terms of utility. These devices are highly beneficial for the patients who require continuous monitoring [9], even if they are not admitted to hospital. Presently, a lot of research is under process to understand the impact of the exposure of electromagnetic radiation on human body [10]. Other limitation of using RF based sensors is interference with other devices present in its vicinity. Therefore, secure and safer communication system for healthcare applications needed nowadays. Visible Light Communication (VLC) is an emerging area that can be used as a medium of wireless communication. Visible light communication has proven to be an efficient technology for data transmission and support intelligent communication system. It has several advantages like high switching speed, better SNR, improved electromagnetic compatibility and less electrical power requirement [11]. This connects light in visible range to networks and is non-hazardous to health.

Visible Light Communication (VLC) sources are able to produce large intensities and therefore have high SNR value. Visible light Communication system provides improved EMC (electromagnetic compatibility) as compared with the RF technology because optical sources does not obstruct with the other existing sources. VLC systems can also be used in sensitive areas such as in hospitals, chemical industries etc that have strict EMC limitations.

In proposed patient monitoring system, common sensors are placed on patient body for healthcare monitoring as shown in Fig. 1 [12]. An Electrocardiogram (ECG) sensor is placed on the chest and a $\mathrm{SpO} 2$ sensor (pulse oximeter) is clamped on the patient index finger to determine level of oxygen in blood. Implementation of patient monitoring system is shown in the Fig. 2 [13]. Sensor reads data from the patient , then this data is being processed by either a Microcontroller Unit (MCU) or Digital Signal Processor (DSP) before transmitted by a wireless transceiver. On the server side, the wireless transceiver receives the patient data wirelessly and then, it process the received data using either MCU or DSP before it is collected in the server. 


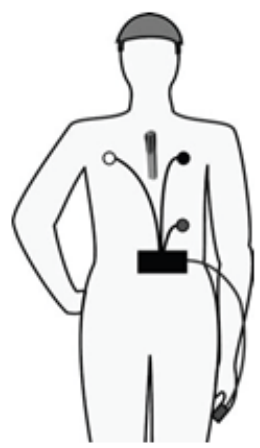

Fig. 1. Patient with biomedical sensors on their body.

Visible Light Communication technolgy uses three different configurations for their transmission; i) Directed Line of Sight (LOS), ii) Non-directed LOS (NLOS) and iii) Non-directed Non-LOS. Depending on the coverage area, mobility, bit rate a specific link configurations can be selected.

i) Directed Line-Of-Sight (LOS): This link configuration use directional source which focuses on optical signals in a very restricted beam. Higher data rates, no multipath distortion, low power required at the source level and are the merits of these configurations.

ii) Non Directed LOS (NLOS): This link configuration wants arrangement between source and receiver side. Higher transmission power is required for these arrangements and these can also suffer from ISI because of multipath propagation that increases according with the coverage area.

iii) Non-directed non-LOS Configuration: This configuration also known as diffused configuration. For mobile and indoor applications this arrangement is mainly used.

Due to ease in implementational, Directed Line Of Sight (LOS) propagation, is implied in proposed system. Medical sensors are placed on patient body and in the base station of hospital,VLC is used to ensure communication between a different transmitters and makes a connection Different mobility scenerios equivalent to the patient moving in hospital room are also proposed in patient monitoring healthcare system. The first mobilty scenerio is in which transmitter height is fixed and in second scenerio transmitter height variations are considered.

In this patient monitoring system,maintaining security and reliability of data is important [14]. Most of the applications currently utlize radio frequency spectrum, leading to highly congested networks, further it is not safe for human health as well as highly vulnerable for hacking. [15]. Visible Light Communication (VLC) is introduced as a more secure and greener alternative with free spectrum, as contrasted to these RF technologies in terms of less congestion and safety issues[16]. Visible light Communication (VLC) system uses LED as transmitters and receivers. VLC allows mechanism to built a wireless adhoc communication system with lowbit-rate (LBR) for

short range communication [17]. VLC systems are highly suited for closed systems like hospitals. Under short range, VLC systems are perfect networks to send high quality signals with the minimum possibility of diffraction of refrection.

For getting biomedical data and health related information onto a portable device, downlink data transmission is done by using VLC system[18]. As light cannot pass through walls, makes VLC more secure against hacking and less hazardous to human health. A complete healthcare system supports both uplink and downlink data transmission but primarily for efficient patient monitoring system uplink data transmission is more important $[17,18]$.

\section{Architecture Of Typical Vlc Based Patient Monitoring System}

Visible Light Communication (VLC) can also suffer from ambient noises coming from other illumination sources which is exposed to the photo detector; although, the Signal-to-Noise Ratio (SNR) can still be acceptable range if proper color filtering is used. VLC photo detector can be directed away from the interference sources since the diffused indirect reflection of visible light is not as strong as IR. Therefore, for VLC, it is still possible to use a simple modulation scheme like OOK modulation scheme. VLC is able to utilize multiple colors in multi-users transmission scenario. Since VLC does not have many noise sources as IR, therefore, lower power transmission can be maintained in VLC for the indoor use. 

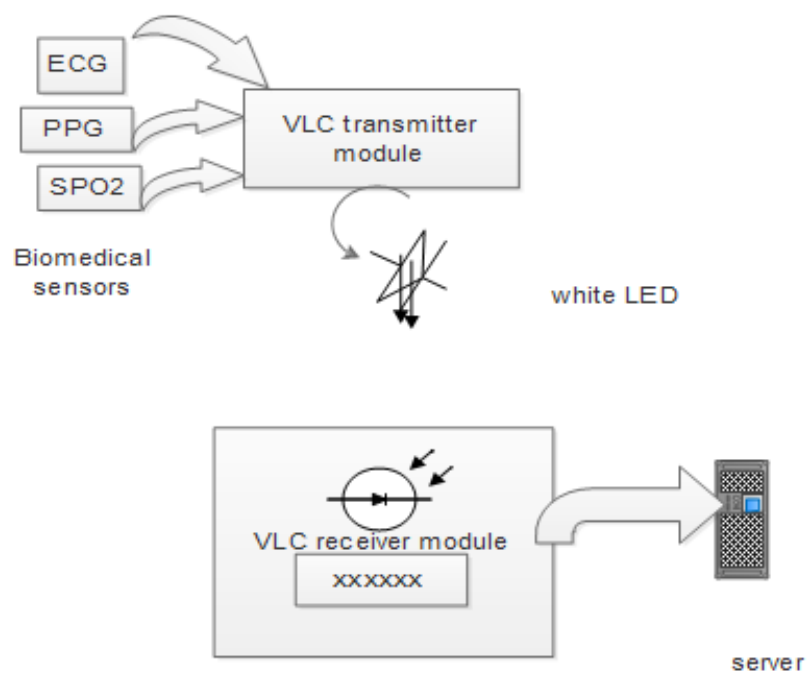

Fig2. Experimental setup for indoor VLC system

LEDs are energy efficient devices and exhibit power saving characteristics, therefore they are preferred to be used in commercial places like Hospitals and healthcare cemters. An experimental setup of VLC system is shown in Fig 2. Here white LED lamps have been used for the transmission of medical data.These emitter are fixed on VLC transmitter module on a printed circuit board.

Modulation schemes that used in visible light communication system are single carrier modulation schemes, multi-carrier modulation schemes and color domain modulation scheme [19].

Single-carrier modulation schemes: In this modulation scheme for communication sinusoidal waves are used. This scheme is suitable for low-to-moderate signal rates. Two mostly used single carrier modulation scheme for visible light communication system are On-off keying (OOK) and Pulse position modulation (PPM). The places where no multipath distortion present, there pulse position modulation scheme are used and it also offers higher bandwidth and are more power efficient. OOK provides a good exchange between performance and complexity.

Multi-carrier modulation schemes: This modulation scheme provides higher data rates. Orthogonal frequency-division multiplexing (OFDM), is the widely used multi-carrier modulation scheme in VLC system. It offers parallel data transmission by sending orthogonal signals between source and receiver side. Thus, it becomes a good alternative in order to expand the more VLC applications.

Color domain modulation schemes: Particularly for visible light communication system, Color domain modulation schemes are widely used. In order to avoid flickering, the total intensity of the output is not varied in color shift keying (CSK).The frequently changing colors seems white to a human eye, however all color channel can be independently detected by optical receiver.

In our proposed healthcare system we widely used OOK modulation schemes for the ease of implementation. The VLC circuit consists of an on-off keying (OOK) non-return to zero (NRZ) modulation circuit which produces a carrier signal. The simplest form of band pass data modulation is OOK a form of amplitude shift keying (ASK). The main advantage of using NRZ over other formats, is that the bandwidth used by this signal is around half of that used in the RZ format. In the circuit, VLC receiver module is interfaced with the microcontroller in order to get desired information . Received data signal is then amplified and demodulated. A microcontroller display device is connected with VLC receiver module circuit to display the received information which is connected to VLC receiver module.

\section{Proposed Vlc Healthcare System}

Proposed patient monitoring system uses white LEDs for VLC based data transmission system .These LEDs can be used for illumination and for data transmission of sensors for static patient in bed. It is considered that usage of illumination LED light will allow the easy control for downlink. A typical patient under monitoring would be static most of the time, therefore, primarily a static model is chosen. A LED unit for data transmission is to be installed for each sensor using a precise transmission module which points upward to the ceiling where the receiver is placed. The transmission modules placed on the patient will have the LOS connection to the photo detectors which is placed on the ceiling. Multiple photodetectors is used to achieve 
diversity effect. Appropriate spacing between photo detectors is required for robustness on the data response.The proposed healthcare system for mobile patient may also be configured for a patient moving in a restricted environment. In a hospital room, environment is considred as a free from each obstacles.

The proposed healthcare system for patient monitoring uses three biomedical signals obtained through sensors for healthcare centers, i.e., ECG, body temperature, Photoplethysmogram (PPG), amount of oxygenated hemoglobin (SPO2) in the blood and pulse ratewhich are transmitted through the LEDs using the OOK modulation. For low data rate OOK is sufficient for this purpose. Based on the predetermined header, the received data is then demodulated and multiplexed. Patient related data is delivered regularly for monitoring purpose, thus both accuracy and reliability becomes an essential part of this system.

\section{Conclusion}

Commercial white LEDs can be used as a clean device for the transmission of clinnical healthcare text data and analog physiological signals.. A typical patient monitoring system using visible light data transmission is presented, the system features multiple patient data transmission continiously using the space diversity of photodetectors. The simple OOK modulation with a low data rate is adequate for transmission of the sensor data and properly placed photodetectors for minimal interference from ambient illumination. The systems is primarily designed bu using three biomedical sensor for healthcare monitoring system.

\section{Reference}

[1]. S. Movassaghi, M. Abolhasan, J. Lipman, D. Smith, and A. Jamalipour, "Wireless body area networks: a survey," IEEECommunications Surveys \& Tutorials, vol. 16, no. 3, pp. 1658-1686, Third Quarter 2014.

[2]. G. V. Crosby, T. Ghosh, R. Murimi, and C. A. Chin, "Wireless body area networks for healthcare: a survey," Int. Journal of Ad hoc,Sensor\& Ubiquitous Computing (IJASUC), vol. 3, no. 3, pp. 1-26, June 2012.

[3]. P. S. Hall and Y. Hao, Antennas and Propagation for Body-Centric Wireless Communications, 2nd P. S. Hall and Y. Hao, Antennas and Propagation for Body-Centric Wireless Communications.

[4]. R. Di Bari, Q. H. Abbasi, A. Alomainy and Y. Hao, "An Advanced Ultra Wideband Channel Model for Body-Centric Wireless Networks", Progress In Electromagnetic Research (PIER), Vol. 136, pp. 79-99, 2013.

[5]. M. M. Khan, Q. H. Abbasi, A. Alomainy and Y. Hao, "Study of Line of- Sight (LoS) and Non-Line-of-Sight (NLoS) Ultra Wideband Off-Body Radio Propagation for Body Centric Wireless Communications in Indoor", in Proceedings of the 5th IEEE European Conference onAntennas and Propagation (EUCAP), April 11-15, 2011, Rome, Italy, pp. 110-114.

[6]. D. K. Cho, S. H. Lee, A. Chang, T. Massey, C.W. Chang, M. H. Tsai, M. Sarrafzadeh, and M. Gerla, "Opportunistic medical monitoring using Bluetooth P2P networks," Int. Symp. on a World of Wireless,Mobile and Multimedia Netw., Newport Beach, CA, pp. 1-6, June 23-26, 2008.

[7]. J. H. Kim, B. G. Choi, J. Heo, and C. S. Hong, "Development of ZigBee mobile router for supporting network mobility in healthcare system," Digest of Technical Papers Intern. Conf. on ConsumerElectronics (ICCE), Las Vegas, NV, pp. 1-2, January 10$14,2009$.

[8]. E. Georgakakis, S. A. Nikolidakis, D. D. Vergados, and C. Douligeris, "An analysis of Bluetooth, Zigbee and Bluetooth LowEnergy and their use in WBANs," in Wireless Mobile Communication and Healthcare, pp. 168-175, Piraus: Springer Berlin Heidelberg, 2011.

[9]. Z. Li, G. Feng, F. Liu, J. Q. Dong, R. Kamoua, and W. Tang, "Wireless health monitoring system," Applications and TechnologyConference (LISAT), pp. 1-4, May 7, 2010.

[10]. 2016Review: Radiofrequency Health, BC Centre for Disease Control. Environmental Health Services.

[11]. D. K. Borah, A. C. Boucouvalas, C. C. Davis, S. Hranilovic, and K. Yiannopoulos, "A review of communication-oriented optical wireless systems," EURASIP J. Wireless Commun. Netw., vol. 2012, no. 1, pp. 91:1-91:28, Mar. 2012

[12]. R. Kitchen, "Effects of radio frequency radiation," in RF and Microwave Radiation Safety, 2nd Ed., Oxford: Newness, 2001.

[13]. A. Jovicic, L. Junyi, and T. Richardson, "Visible light communication: opportunities, challenges and the path to market,"IEEE Communication Magazine, vol. 51, no. 12, pp. 26-32, December 2013.

[14]. S. Schmid, G. Corbellini, S. Mangold, and T. Gross. An LED to LED Visible Light Communication System with software-based synchronization, in the IEEE Optical Wireless Communication Globecom Workshops, pp. 1264-1268, December 2012.

[15]. X. W. Ng, and W.Y. Chung, "VLC based medical healthcare information system," Biomed. Eng. Appl. Basis Communication, vol. 24 , no. 2 , p. $155,2012$.

[16]. M.J. Field and J. Grigsby, "Telemedicine and remote patient monitoring," JAMA, 2002; vol. 288, no. 4, pp. 423-425, July 24,2002.

[17]. S. S. Torkestani, S. Sahuguede, A. J. Vergonjanne, J. Cances, and J. C. Daviet, "Infrared communication technology applied to indoor mobile healthcare monitoring system," International Journal of EHealthand Medical Communications (IJEHMC), vol. 3, no. 3, pp. 1- 11, July-September 2012

[18]. Sumita Mishra, Sachin Kumar, Pallavi Asthana, Anil Kumar, Nidhi Mathur, "Short Range Visible Light Communication: Enabling Technology, Challenges and Applications". 